\title{
Cilostazol Added to Dual Antiplatelet Therapy for Patients with High Risk of Restenosis after Drug-Eluting Stent Implantation: A Systematic Review and Meta-Analysis of RCTs
}

Hai-Bin Chen, Xinlu Zhang, Hongbin Liang, Xuewei Liu and Jiancheng Xiu*

Department of Cardiology, Nanfang Hospital, Southern Medical University, Guangzhou, Guangdong, China

\begin{abstract}
Background: Cilostazol added to Dual Antiplatelet Therapy (DAT: aspirin, clopidogrel) reduces revascularization without increased bleeding in patients after Drug-Eluting Stent (DES) implantation. However, doubts remain about which patients benefit most from cilostazol-based Triple Antiplatelet Therapy (TAT) after DES.

Materials and Results: PubMed, EMBASE, CENTRAL databases were systematically searched. Randomized Controlled Trials (RCTs) comparing TAT and DAT for patients with high risk of restenosis (defined as obesity, diabetes, and long and/or multivessel coronary lesions) were included. Five RCTs were included, involving 2442 patients. The TAT group showed a significant reduction in MACEs (4.16\% vs. $8.86 \%, \mathrm{RR}: 0.47,95 \% \mathrm{Cl}: 0.32$ to $0.68, p<0.001)$, in-stent late loss $(0.34$ vs. 0.46 , SMD: $-0.22,95 \% \mathrm{Cl}:-0.32$ to $-0.11, p<0.001)$, TVR $(3.36 \%$ vs. $6.80 \%$, RR: 0.49 , $95 \% \mathrm{Cl}: 0.34$ to $0.71, p<0.001)$, and in-stent restenosis ( $6.86 \%$ vs. $11.45 \%, \mathrm{RR}: 0.60,95 \% \mathrm{Cl}: 0.43$ to $0.84, p=0.003)$ compared with the DAT group. There was no difference in all-cause mortality $(1.56 \%$ vs. $0.82 \%, \mathrm{RR}: 1.82,95 \% \mathrm{Cl}$ : 0.87 to $3.77, p=0.110)$, bleeding ( $3.52 \%$ vs. $3.28 \%$, RR: $1.07,95 \% \mathrm{Cl}: 0.71$ to $1.63, \mathrm{p}=0.745)$ and stent thrombosis ( $0.82 \%$ vs. $0.66 \%$, RR: $1.4,95 \% \mathrm{Cl}: 0.50$ to $3.06, p=0.641$ ) between the two groups, whereas the incidence of other adverse reactions ( $11.38 \%$ vs. $6.39 \%$, RR: $1.78,95 \% \mathrm{Cl}: 1.37$ to $2.33, p<0.001)$ and drug discontinuation $(16.29 \%$ vs. $5.15 \%$, RR: $4.60,95 \% \mathrm{Cl}: 1.24$ to $17.08, p=0.023$ ) was greater in the TAT group than in the DAT group.
\end{abstract}

Conclusions: Compared with DAT, patients with a high risk of restenosis benefited from TAT in reduced stent restenosis and revascularization after DES implantation, without increases in all-cause mortality and bleeding, but accompanied by a higher incidence of other adverse reactions and drug discontinuation.

Keywords: Cilostazol; Triple antiplatelet therapy; Dual antiplatelet therapy; Drug-eluting stent

\section{Introduction}

The implantation of Drug-Eluting Stents (DES) implantation and Dual Antiplatelet Therapy (DAT: aspirin, clopidogrel) are indispensable treatments for patients undergoing Percutaneous Coronary Intervention (PCI). However, this treatment combination carries a risk of stent thrombosis and restenosis [1-3], which is associated with unresponsiveness to clopidogrel (clopidogrel resistance) [4,5] and High on-Treatment Platelet Reactivity (HTPR) $[3,6]$. Although DES reduces the incidence of restenosis and repeat revascularization compared to Bare-Metal Stents (BMS), DES-associated restenosis and clinical events remain notable clinical problems, especially in patients with long and/ or multivessel coronary lesions [7], obesity [8,9] or diabetes [10].

Cilostazol is a selective and reversible inhibitor of phosphodiesterase III that is primarily used to relieve intermittent claudication due to its antiplatelet and vasodilating effects [11]. In vitro, the drug affects different cell lines, including endothelial cells, vascular smooth muscular cells, and platelets, by augmenting intraplatelet cyclic adenosine monophosphate levels [12,13]. Furthermore, cilostazol exerts pleiotropic effects, such as inhibiting the progression of carotid atherosclerosis [14].

Recently, several meta-analyses of Randomized Controlled Trials (RCTs) have reported a benefit of TAT for long-term mortality and reducing revascularization without increased bleeding in patients undergoing PCI [15-17]. However, which patients benefit most from TAT after DES implantation, in terms of restenosis, remains unknown. Although several RCTs [18-20] have suggested that TAT benefits patients with a high risk of restenosis after DES implantation, those involved small numbers of patients, and the data are inconsistent. To investigate the effects of TAT in patients with a high risk of restenosis after DES implantation, we conducted a meta-analysis of RCTs.

\section{Methods}

\section{Data sources and searches}

We systematically searched all published studies that compared DAT, with and without cilostazol, in patients with a high risk of restenosis with DES (including those with obesity, diabetes, and long and/or multivessel coronary lesions) using the Cochrane Central Register of Controlled Trials (CENTRAL), PubMed and Embase. The literature search was updated on 31 October 2014 during the final revision process without any language restriction using the medical subject headings and text words, such as "cilostazol", "dual antiplatelet therapy", "triple antiplatelet therapy", "stent implantation", "drugeluting stent", "stent restenosis", "diabetes", "obese", "long lesions", "multivessel lesions", and "randomized controlled trials". In addition

*Corresponding author: Jiancheng Xiu, Department of Cardiology, Nanfang Hospital, Southern Medical University, Guangzhou, Guangdong, 510515, China, Tel: +62787390; E-mail: xiujc@126.com

Received: February 13, 2015; Accepted: March 10, 2015; Published: March 15, 2015

Citation: Chen HB, Zhang X, Liang H, Liu X, Xiu J (2015) Cilostazol Added to Dual Antiplatelet Therapy for Patients with High Risk of Restenosis after Drug-Eluting Stent Implantation: A Systematic Review and Meta-Analysis of RCTs. Cardiol Pharmacol 4: 133. doi:10.4172/2329-6607.1000133

Copyright: () 2015 Chen HB et al. This is an open-access article distributed under the terms of the Creative Commons Attribution License, which permits unrestricted use, distribution, and reproduction in any medium, provided the original author and source are credited. 
to searching databases, the reference lists of all the identified studies were manually searched. Internet-based sources of information (Google Scholar, Microsoft Academic Search, Web of Knowledge and www.clinicaltrial.gov) were also searched for more trials.

\section{Study selection}

Two reviewers (HB.C. and X. Z.) independently performed study selection and assessed the eligibility of RCTs identified by searching the relevant databases, with disagreements resolved by consensus or by the opinion of a third reviewer (J. X.), if necessary. Studies were considered for inclusion if they were RCTs involving patients with a high risk of restenosis after DES implantation (including those with obesity, diabetes, and long and/or multivessel lesions) and randomly assigned patients to TAT and DAT groups. Additional studies were evaluated if they included at least one of the following interesting outcomes: allcause death, MACEs, TVR, TLR, stent restenosis, in-stent late loss, insegment late loss, stent thrombosis, bleeding, other adverse reactions, and drug discontinuation.

\section{Data extraction and quality assessment}

The data were extracted and presented according to the Providing Innovative Service Models and Assessment (PRISMA) criteria [21]. Two reviewers (HB.C. and X. Z.) independently extracted data from all the included studies and assessed the data quality, with disagreements resolved by consensus or by the opinion of a third reviewer (J. X.), if necessary. The extracted data were first author, the year of publication, country, follow-up duration, number of participants and drug protocol, the pooled efficacy outcomes, including all-cause death, MACEs, in-stent late loss, in-segment late loss, stent restenosis, stent thrombosis, TVR, TLR, bleeding, and other adverse reactions (such as rash, gastrointestinal trouble, thrombocytopenia, neutropenia, hepatic dysfunction, or headache) and drug discontinuation during the treatment period. The authors may be contacted by e-mail for more detailed information.

The study methodology quality assessment was undertaken by a validated scale (modified Jadad score, according to the Jadad scoring method) [22] based on the following items: randomization, concealment of allocation, double blinding, withdrawals and dropouts. A score of 1 point was given for each item satisfied, and 1 additional point was given for high quality of randomization, concealment and double blinding. A high quality study was defined by a score greater than three.

\section{Outcomes definitions}

We accepted original trial definitions for all outcomes. Major efficacy outcomes were all-cause mortality, MACEs and bleeding. Minor efficacy outcomes were stent thrombosis, in-stent late loss, insegment late loss, TLR, TVR, binary angiographic restenosis (stenosis diameter $>50 \%$ ) both in-stent and in-segment (stented segment and margins $5 \mathrm{~mm}$ proximal and distal to the stent), other adverse reactions and drug discontinuation.

\section{Statistical analyses}

Review-Manager software (RevMan, version 5.2 for Windows) and STATA12.0 software (STATA Corp. College Station, TX, USA) were used to perform this analysis. We determined the pooled Standard Mean Difference (SMD) and corresponding 95\% Confidence Intervals (CI) for the continuous data and pooled Risk Ratios (RR) for the dichotomous data.
The heterogeneity of the data was assessed visually by L'Abbe plots [23] and Galbraith plots [24]. The Q test and $I^{2}$ statistic were also used to evaluate heterogeneity between studies, and we considered a $p$ value less than 0.1 as statistically significant. A fixed-effects model (MantelHaenszel method) was used for analysis in the case of low or moderate heterogeneity among trials $\left(I^{2} \leq 50 \%, p<0.1\right)$. If there indicated high heterogeneity $\left(I^{2} \geq 50 \%, p<0.1\right)$, we tried to find clinical heterogeneity across the studies first and addressed it by sensitivity analysis meanwhile, a random-effect model (DerSimonian-Laird method) was used.

Publication bias was estimated by the use of funnel plots, as well as Begg's rank correlation test [25] and Egger's linear regression test [26]. A value of $p \leq 0.05$ (two-sided) was considered to be statistically significant. If publication bias seemed to be present through this process, the Duval and Tweedie's trim-and-fill [27] procedure was used to estimate the possible impact of unpublished studies on the pooled estimate.

\section{Results}

\section{Eligible studies}

A total of 127 potential relevant articles were retrieved by database and manual searching. Forty-eight duplicates and 50 irrelevant articles were excluded by title and abstract assessment. Twenty-four articles were excluded for not fulfilling our inclusion criteria after a more detailed assessment. As a result, five RCTs that met the inclusion criteria were included in this meta-analysis, involving 2442 patients (TAT: DAT=1221: 1221) $[18,20,28-30]$. The process, with reasons for exclusion, was described in Figure 1.

\section{Study characteristics and study quality}

The baseline characteristics of each RCT were described in Table 1. All five RCTs were conducted in Asia (four RCTs in South Korea and one RCT in China) and published in English between 2007 and 2014. The duration of follow-up ranged from 6 to 12 months.

A summary of the quality assessment of each RCTs was shown in Table 2. The methods of randomization and reasons for withdrawals, and dropout were described in all the RCTs. One [18] of the five RCTs was performed with concealed treatment allocation; only DeclareLong II was performed in a double-blinded fashion. According to the modified Jadad score, four RCTs were classified as high quality and 1 RCT was classified as low quality.

\section{Major efficacy outcomes}

All-cause mortality: Five RCTs reported all-cause deaths in the TAT group (1221 patients' total) and the DAT group (1221 patients total). A heterogeneity test showed no statistical evidence of heterogeneity among these studies $\left(I^{2}=0 \%, p=0.501\right)$. Pooled analysis showed no difference between the TAT group and the DAT group in all-cause mortality $(1.56 \%$ vs. $0.82 \%$, RR: $1.82,95 \%$ CI: 0.87 to 3.77 , $p=0.110$ ) (Figure 2A).

MACEs: Four RCTs reported MACEs in the TAT group (with 913 patients) and the DAT group (with 914 patients). A heterogeneity test showed no statistical evidence of heterogeneity among these studies $\left(I^{2}=0 \%, p=0.744\right)$. The pooled analysis showed a significantly lower incidence of MACEs in the TAT group than in the DAT group $(4.16 \%$ vs. $8.86 \%$, RR: $0.47,95 \%$ CI: 0.32 to $0.68, p<0.001$ ) (Figure $2 \mathrm{~B}$ ).

Bleeding: Five RCTs reported bleeding in both groups (with 1221 patients total for each group). The heterogeneity test showed 


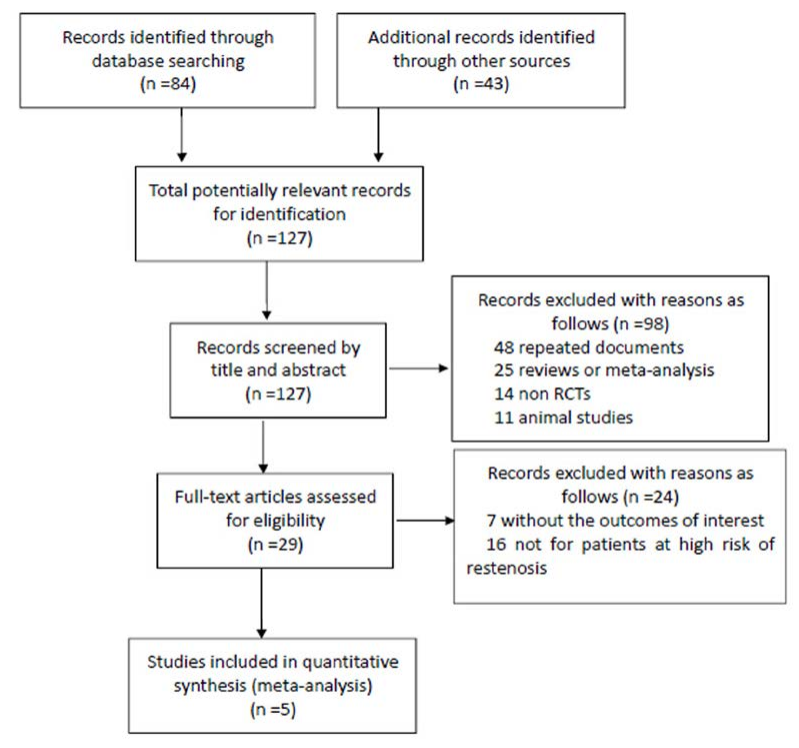

Fig.1 Flow diagram of the trial selection process

Figure 1: Flow diagram of the trial selection process.

no statistical evidence of heterogeneity among these studies $\left(I^{2}=0 \%\right.$, $p=0.803)$. The pooled analysis revealed no difference between the TAT group and the DAT group in bleeding (3.52\% vs. $3.28 \%$, RR: $1.07,95 \%$ CI: 0.71 to $1.63, p=0.745$ ) (Figure $2 \mathrm{C}$ ).

\section{Minor efficacy outcomes}

Stent thrombosis (ST): Five RCTs reported ST in the TAT group (with 1221 patients) and the DAT group (with 1221 patients). The heterogeneity test showed no statistical evidence of heterogeneity among these studies $\left(I^{2}=0 \%, p=0.504\right)$. The pooled analysis showed no difference between the TAT group and the DAT group in ST $(0.82 \%$ vs. $0.66 \%$, RR: $1.4,95 \%$ CI: 0.50 to $3.06, p=0.641$ ) (Figure 2D).

Late loss: Three RCTs reported late loss in both groups, with 700 patients in the TAT group, and 699 patients in the DAT group. The heterogeneity test uncovered no statistical evidence of heterogeneity among these studies $\left(I^{2}=0 \%, p=0.951\right.$ for in-stent late loss and $I^{2}=0 \%$, $p=0.647$ for in-segment late loss). The pooled analysis showed a significant difference between the TAT group and the DAT group in either in-stent late loss $(0.34 \mathrm{~mm}$ vs. $0.46 \mathrm{~mm}$, SMD: $-0.22,95 \% \mathrm{CI}$ : -0.32 to $-0.11, p<0.001)$ or in-segment late loss $(0.36 \mathrm{~mm}$ vs. $0.50 \mathrm{~mm}$, SMD: $-0.29,95 \%$ CI: -0.39 to $-0.18, p<0.001$ ) (Figure 2E1 and Figure 2E2).

TLR: Five RCTs reported TLR in both the TAT group, with 1221 patients, and the DAT group, with 1221 patients. The heterogeneity test showed no statistical evidence of heterogeneity among these studies $\left(I^{2}=0 \%, p=0.944\right)$. The pooled analysis revealed significantly fewer occurrences of TLR in the TAT group than in the DAT group in TLR ( $2.79 \%$ vs. $5.98 \%$, RR: $0.47,95 \%$ CI: 0.31 to $0.69, p<0.001$ ) (Figure $2 \mathrm{~F}$ ).

TVR: Five RCTs reported TLR in the TAT group, with 1221 patients, and the DAT group, with 1221 patients. The heterogeneity test showed no statistical evidence of heterogeneity among these studies $\left(I^{2}=0 \%, p=0.990\right)$. The pooled analysis revealed a significantly lower incidence of TVR in the TAT group than in the DAT group $(3.36 \%$ vs. $6.80 \%$, RR: $0.49,95 \%$ CI: 0.34 to $0.71, p<0.001$ ) (Figure $2 \mathrm{G}$ ).
Binary angiographic restenosis: Three RCTs reported binary angiographic restenosis in the TAT group (700 patients) and DAT group (699 patients). The heterogeneity test showed no statistical evidence of heterogeneity among these studies $\left(I^{2}=0 \%, p=0.734\right.$ for in-stent restenosis and $I^{2}=0 \%, p=0.880$ for in-segment restenosis). The pooled analysis showed significantly fewer reports of in-stent restenosis in the TAT group than in the DAT group (6.86\% vs. $11.45 \%$, RR: 0.60 , 95\% CI: 0.43 to $0.84, p=0.003$ ), with a similar outcome for in-segment restenosis (7.57\% for TAT vs. $13.16 \%$ for DAT, RR: $0.56,95 \%$ CI: 0.42 to $0.80, p=0.001$ ) (Figure $2 \mathrm{H} 1$ and Figure $2 \mathrm{H} 2$ ).

Other adverse reactions: Five RCTs reported other adverse reactions in the TAT group, with 1221 patients, and the DAT group, with 1221 patients. The heterogeneity test showed no statistical evidence of heterogeneity among these studies $\left(I^{2}=20.4 \%, p=0.285\right)$. The pooled analysis showed a significantly higher number of other adverse reactions in the TAT group and in the DAT group $(11.38 \%$ vs. $6.39 \%$, RR: $1.78,95 \%$ CI: 1.37 to 2.33 , p<0.001) (Figure 2I).

\section{Drug discontinuation}

Three RCTs reported drug discontinuation in the TAT group, with 700 patients, and the DAT group, with 699 patients. The heterogeneity test showed statistical evidence of heterogeneity among these studies $\left(I^{2}=87.0 \%, p<0.001\right)$. The pooled analysis uncovered a significantly greater incidence of drug discontinuation in the TAT group than in the DAT group ( $16.29 \%$ vs. $5.15 \%$, RR: $4.60,95 \%$ CI: 1.24 to 17.08 , $p=0.023$ ) (Figure 2J).

\section{Sensitivity analysis and major sources of heterogeneity}

The heterogeneity tests showed significant statistical evidence of heterogeneity only for the drug discontinuation data $\left(I^{2}=87.0 \%\right.$, $p<0.001$ ), and the results of the L'Abbe and Galbraith plots are shown in the supplemental data (Figure S1). The sensitivity analysis of the drug discontinuation data showed the Declare-Diabetes study appeared to be different from the rest of the studies (Figure 3).

\section{Publication bias}

Egger's linear regression test revealed the presence of publication bias only for drug discontinuation ( $p=0.036,95 \%$ CI: 1.485 to 9.007 ) (Table 3). Other outcomes seem to have no publication bias through Begg's rank correlation test and Egger's linear regression test; however, a funnel plot for MACEs, bleeding and ST seemed to be asymmetric (Figure 4). Therefore, the Duval and Tweedie's trim-and-fill procedure, which conservatively imputes hypothetical negative unpublished studies to mirror the positive studies that cause funnel plot asymmetry, was conducted for these endpoints; the results suggest the presence of a publication bias for drug discontinuation (Figure 5).

\section{Discussion}

Despite optimal therapy, such as dual antiplatelet therapy and DES implantation which are recommended in the current guidelines, patients undergoing PCI still suffer a significant risk of restenosis and deleterious clinical events [1,31], especially in patients with obesity, diabetes, and long and/or multivessel coronary lesions [7-10]. Intensified antiplatelet therapy appears to be more important for these high risk patients. Recently, some clinical trials have demonstrated that consolidated antiplatelet therapy could decrease platelet activation and aggregation to give patients a lower incidence of ischemic events $[14,17]$. Several previous meta-analyses of TAT supported the application of TAT in patients with PCI; however, most of them involved RCTs that were limited to relatively low-risk candidates, which may bias effect 
Citation: Chen HB, Zhang X, Liang H, Liu X, Xiu J (2015) Cilostazol Added to Dual Antiplatelet Therapy for Patients with High Risk of Restenosis after Drug-Eluting Stent Implantation: A Systematic Review and Meta-Analysis of RCTs. Cardiol Pharmacol 4: 133. doi:10.4172/2329-6607.1000133

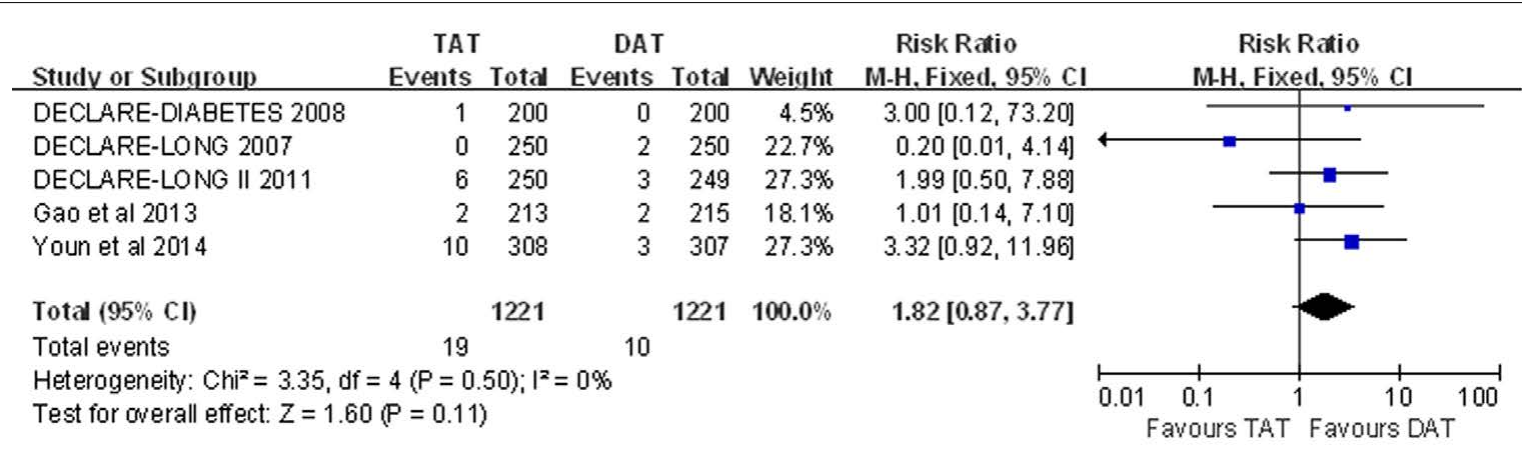

Figure 2: Meta-analysis of efficacy outcomes A: All-cause mortality.

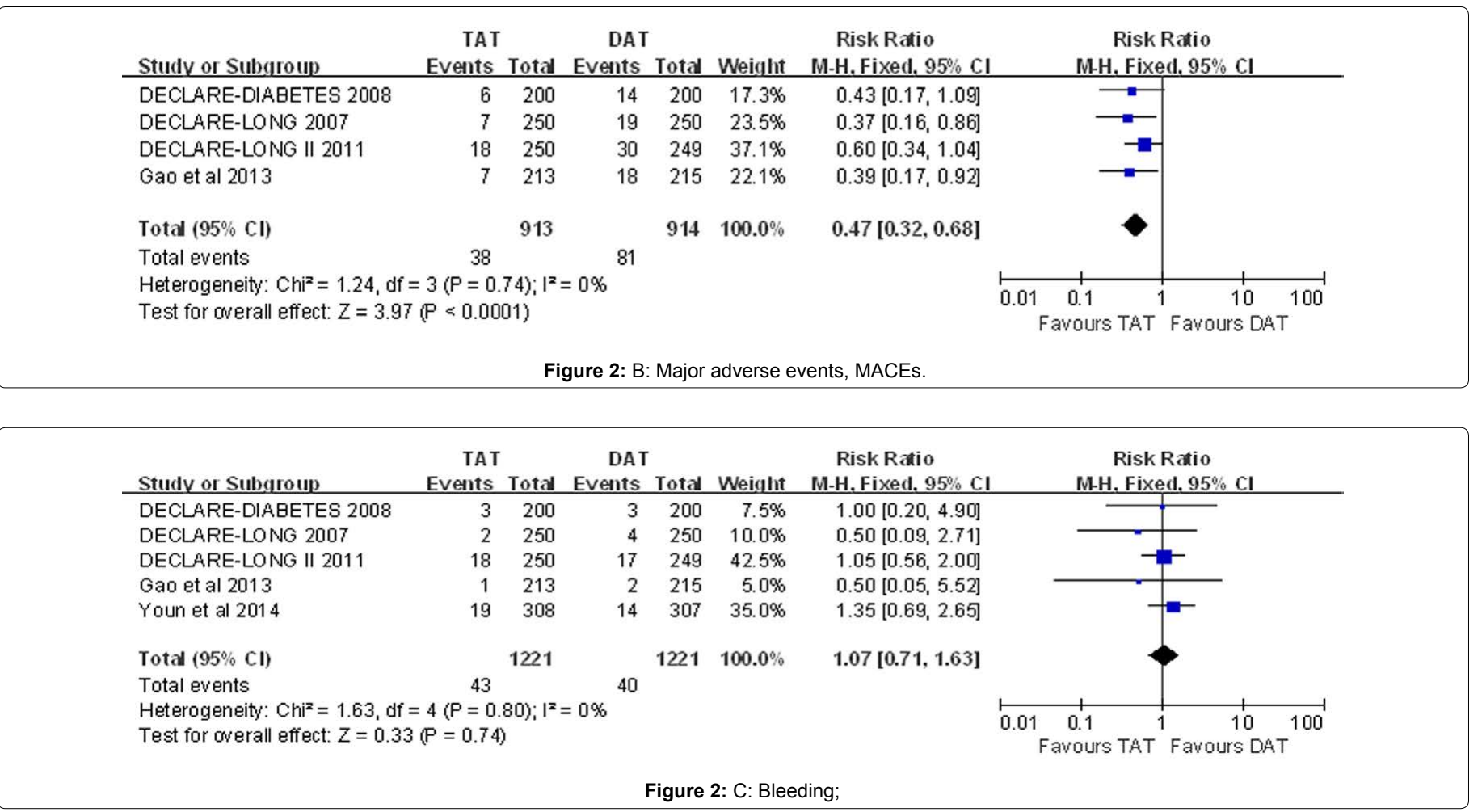

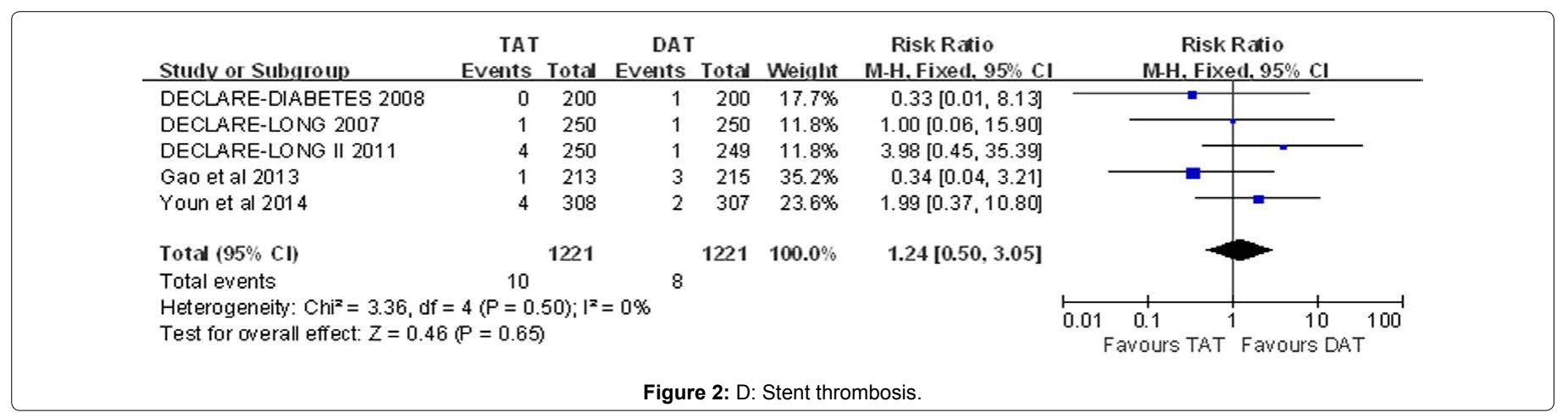


Citation: Chen HB, Zhang X, Liang H, Liu X, Xiu J (2015) Cilostazol Added to Dual Antiplatelet Therapy for Patients with High Risk of Restenosis after Drug-Eluting Stent Implantation: A Systematic Review and Meta-Analysis of RCTs. Cardiol Pharmacol 4: 133. doi:10.4172/2329-6607.1000133

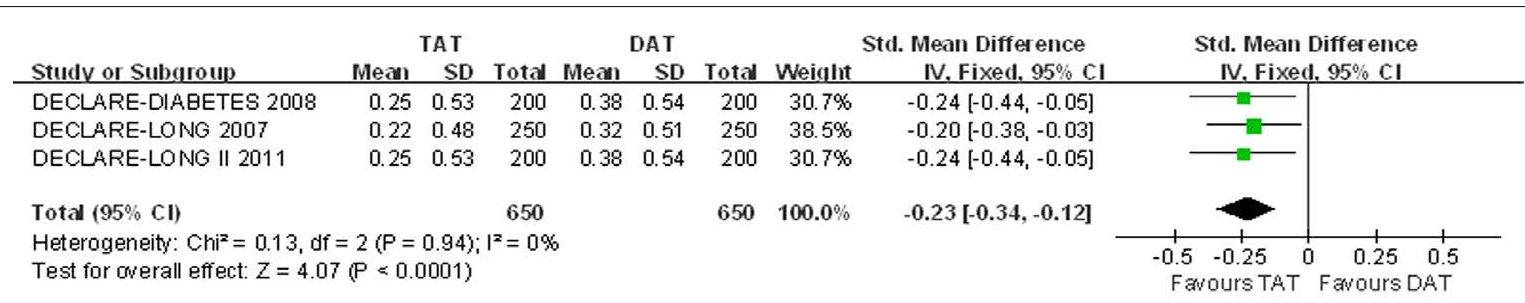

Figure 2: E1: In-stent late loss.

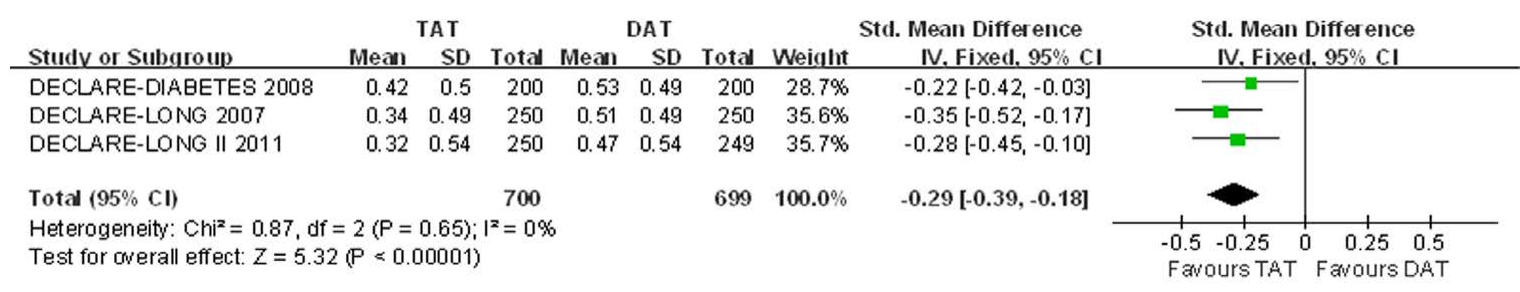

Figure 2: E2: In-segment late loss.

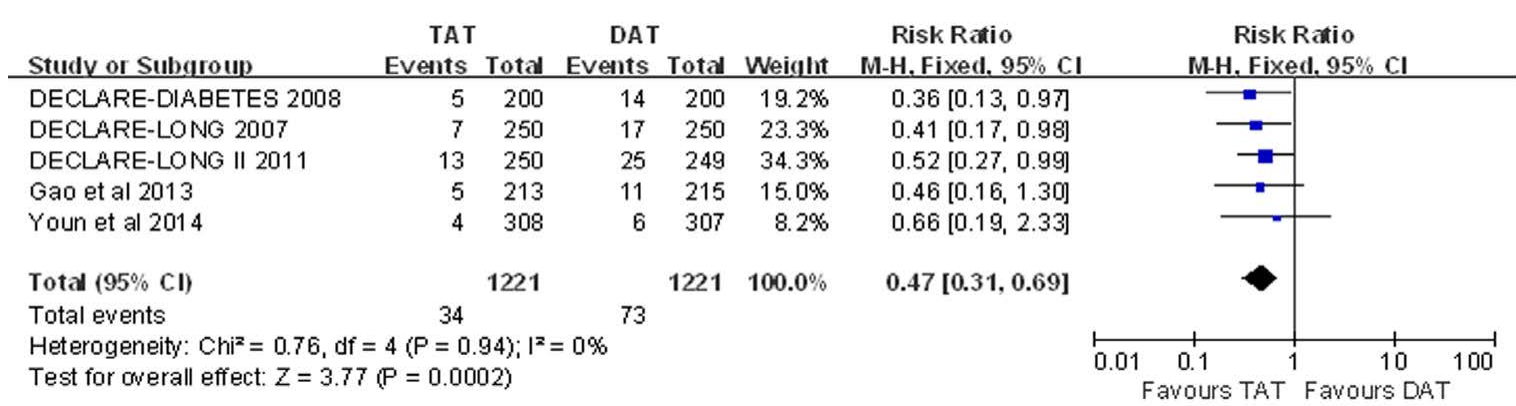

Figure 2: F: Target lesion revascularization, TLR.

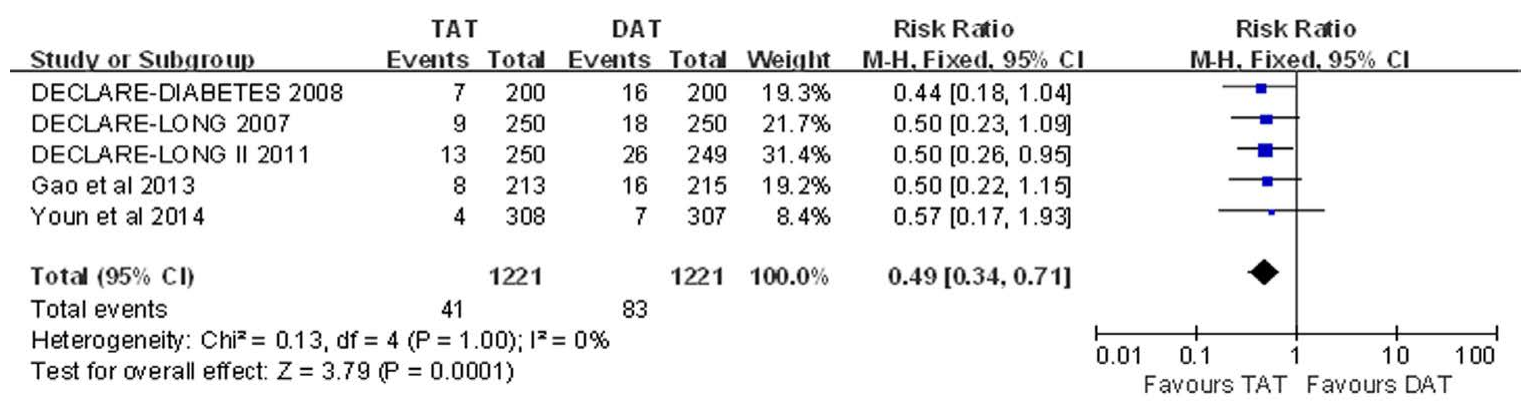

Figure 2: G: Target vessel revascularization, TVR.

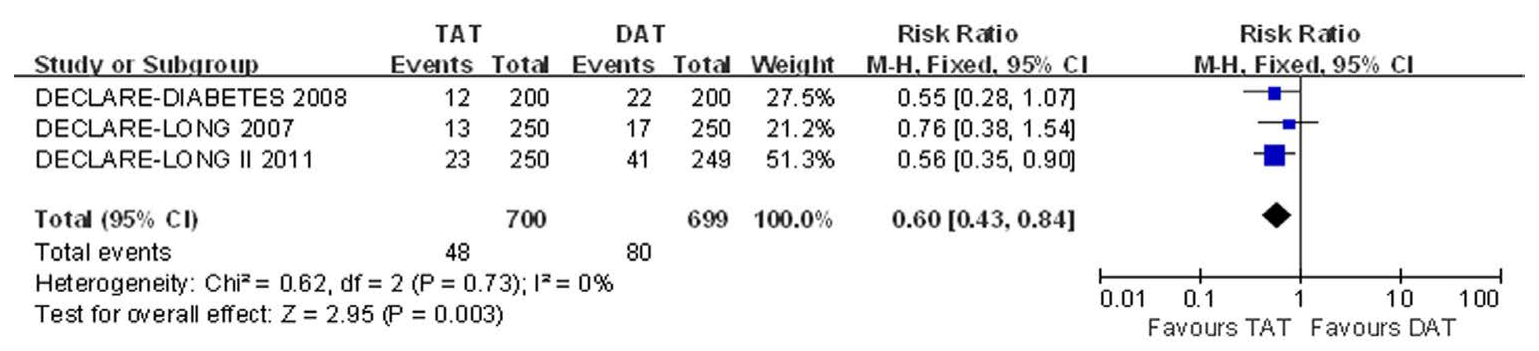

Figure 2: $\mathrm{H} 1$ : In-stent restenosis. 
Citation: Chen HB, Zhang X, Liang H, Liu X, Xiu J (2015) Cilostazol Added to Dual Antiplatelet Therapy for Patients with High Risk of Restenosis after Drug-Eluting Stent Implantation: A Systematic Review and Meta-Analysis of RCTs. Cardiol Pharmacol 4: 133. doi:10.4172/2329-6607.1000133

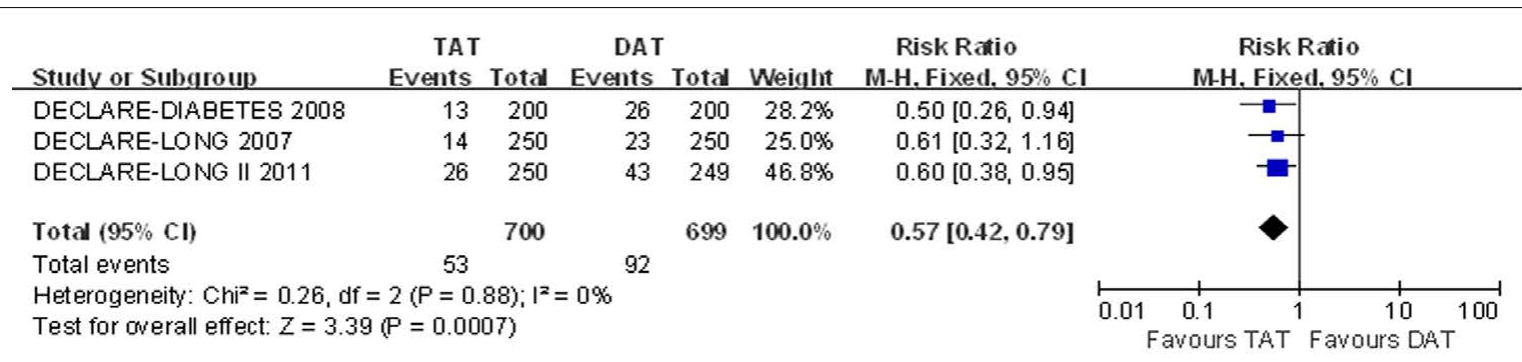

Figure 2: H2: In-segment restenosis.

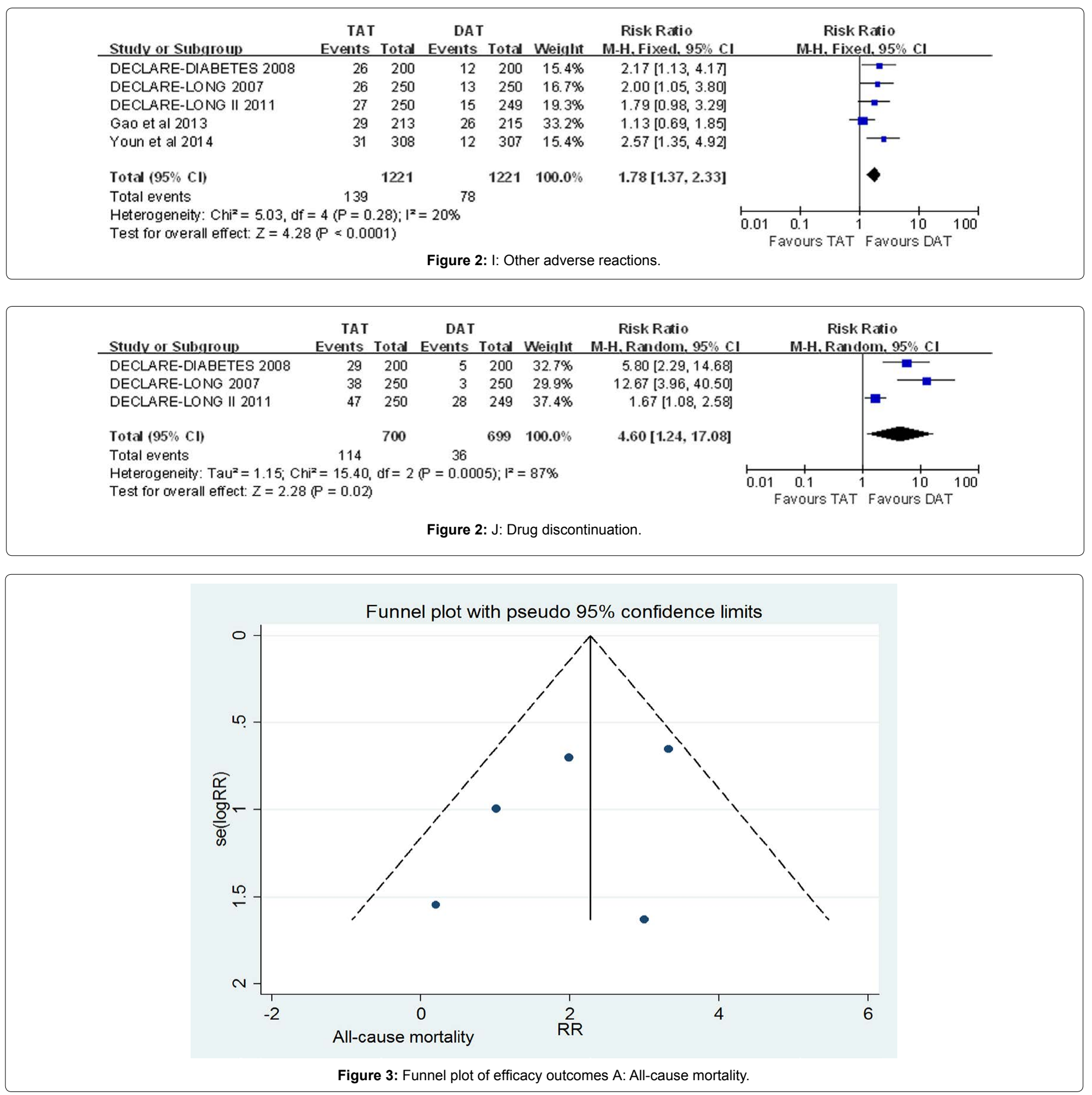




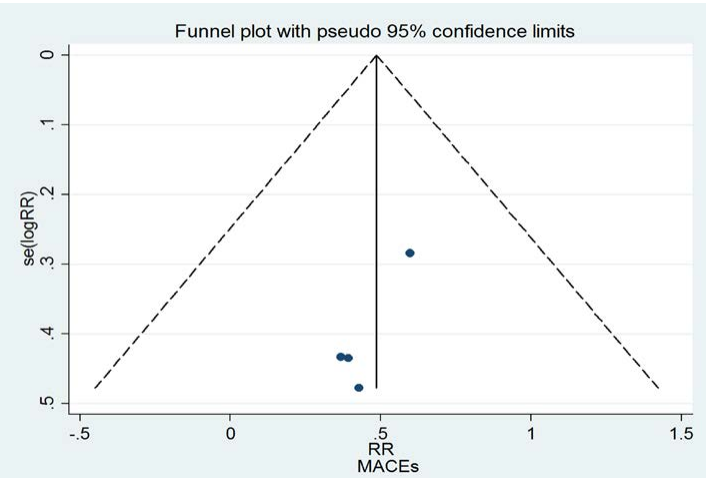

Figure 3: B: Major adverse events, MACEs.

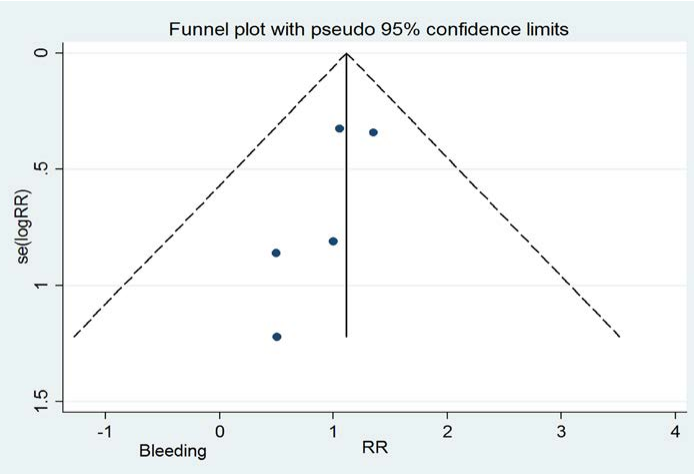

Figure 3:C: Bleeding.

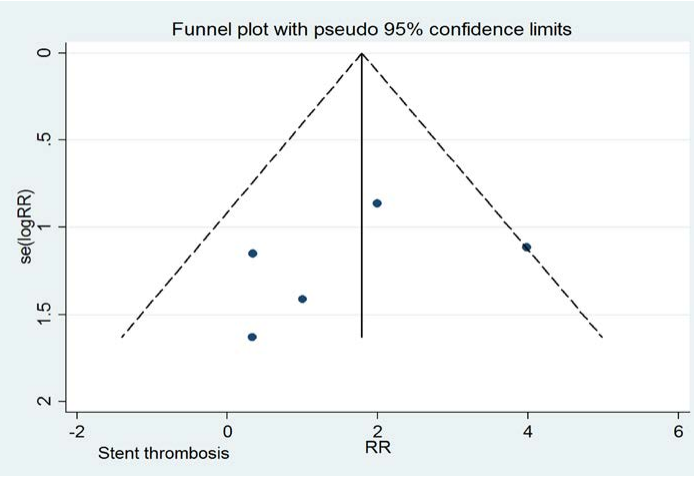

Figure 3: D: Stent thrombosis.

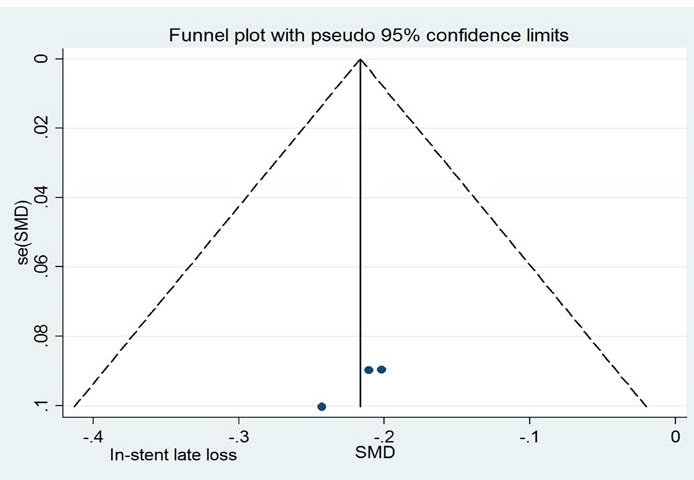

Figure 3: E1: In-stent late loss.

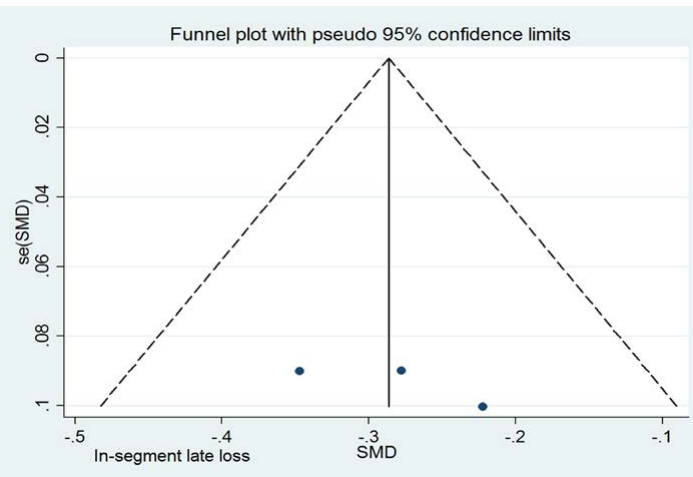

Figure 3: E2: In-segment late loss.

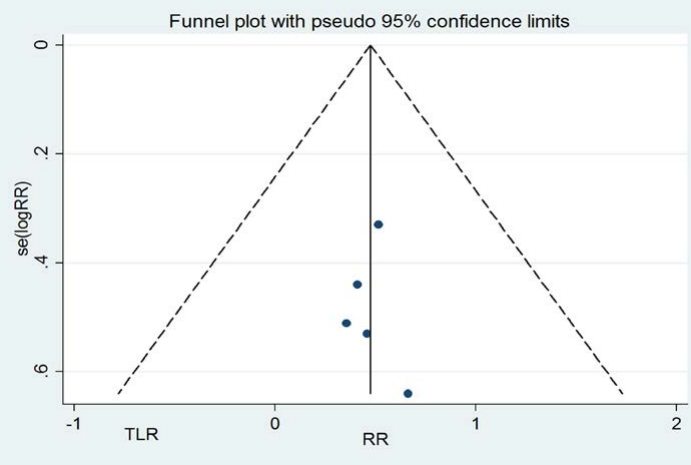

Figure 3: F: Target lesion revascularization, TLR.

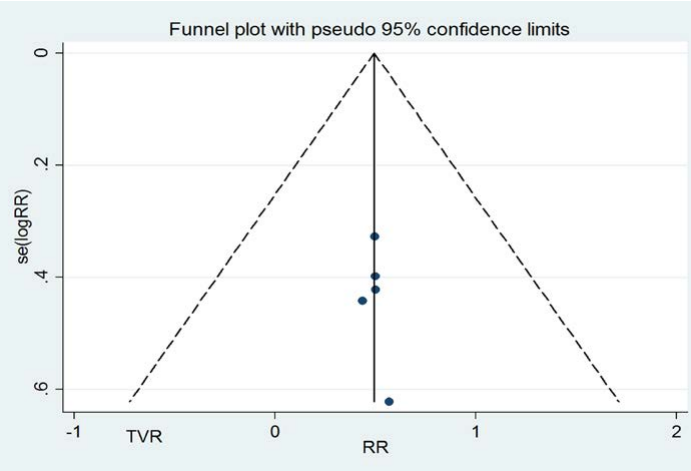

Figure 3: G: Target vessel revascularization, TVR.

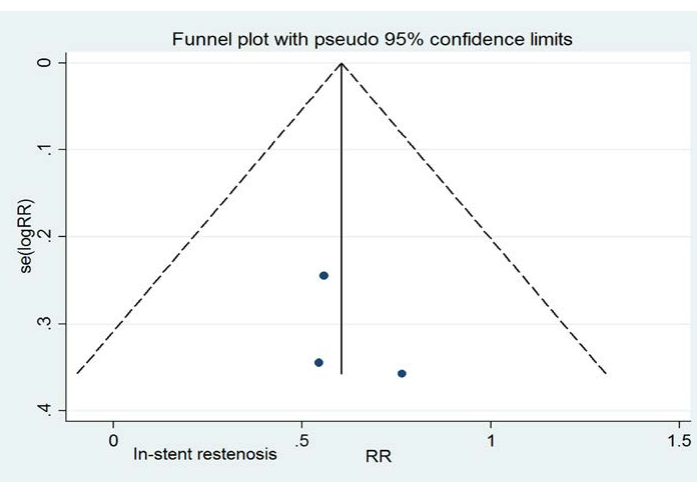

Figure 3: H1: In-stent restenosis. 


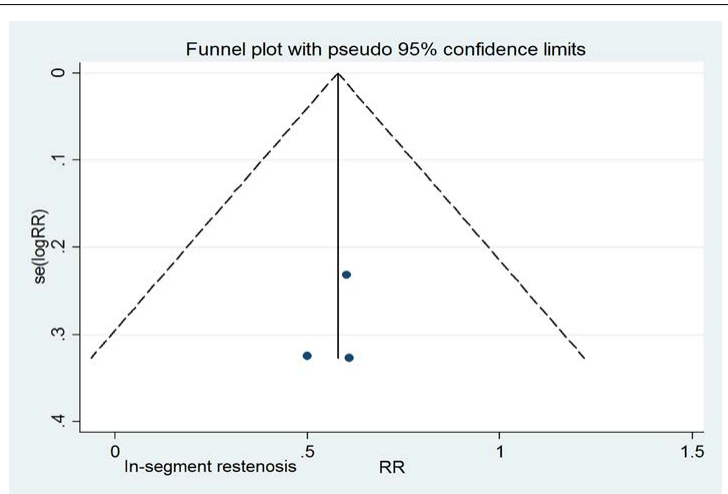

Figure 3: H2: In-segment restenosis

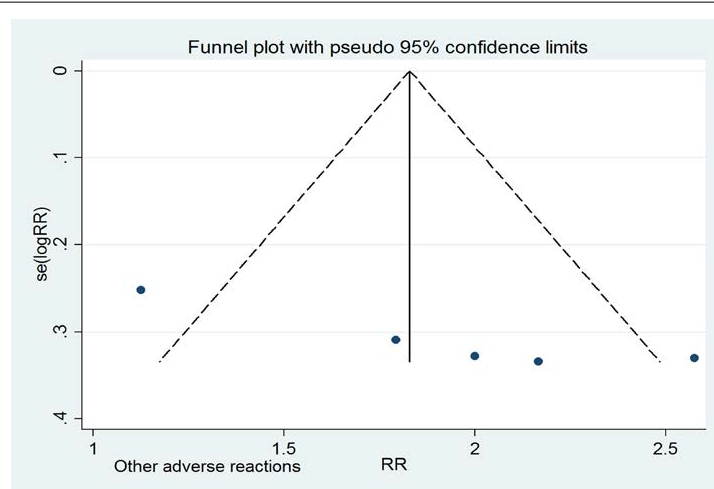

Figure 3: I: Other adverse reactions.

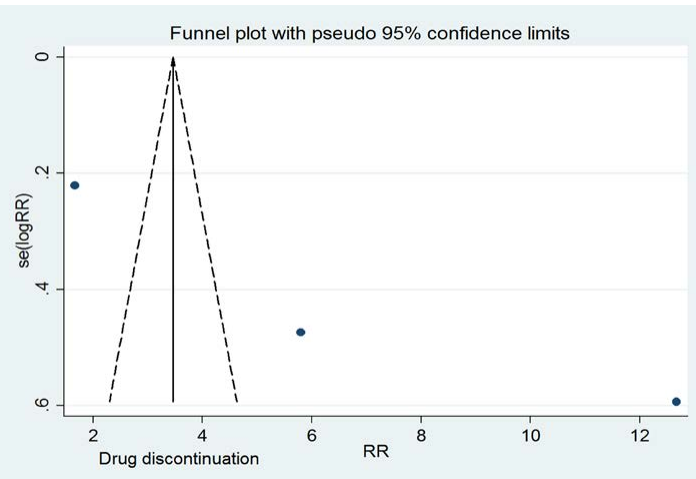

Figure 3: J: Drug discontinuation.

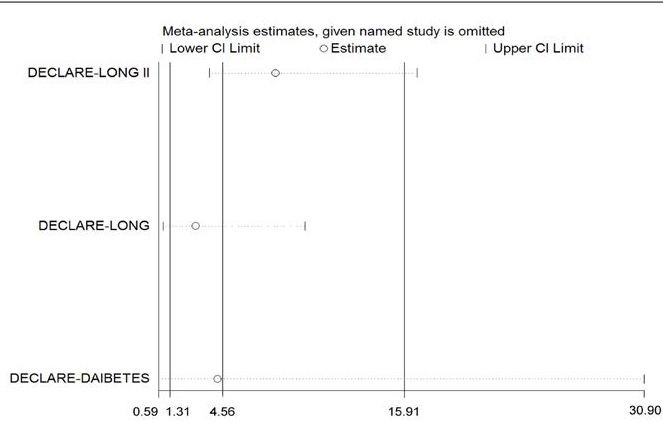

Figure 4: Results of sensitivity analyses for drug discontinuation.

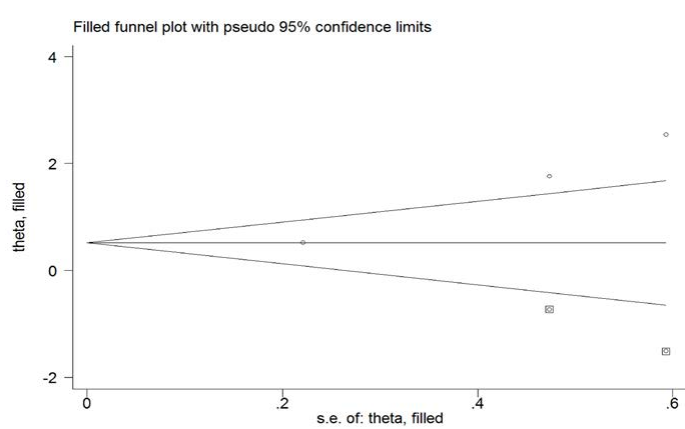

Figure 5: The Duval and Tweedie's trim-and-fill procedure for drug discontinuation. S.E. $=$ standard error

for those with a high risk of restenosis. As no evidence was found for cilostazol therapy in left-main disease, graft-vessel disease, or Chronic Total Occlusion (CTO), we just defined patients at high risk of restenosis after DES implantation as the populations with obesity, diabetes, and long and/or multivessel coronary lesions in this study.

The results from 2242 patients incorporating 5 RCTs also showed benefits of TAT to patients with a high risk of restenosis after DES implantation. The main findings in our meta-analysis were the following: (1) the TAT group was associated with a significantly lower number of occurrences of MACEs, binary angiographic restenosis, late loss of MLD, and incidences of TVR and TLR compared with DAT group; (2) there was no difference between the two groups in bleeding and stent thrombosis; and (3) the incidence of other adverse reactions, such as rash, gastrointestinal trouble, thrombocytopenia, neutropenia, hepatic dysfunction, headache, and drug discontinuation, was higher for the TAT group than the DAT group. These results are consistent with previous meta-analyses.

As a phosphodiesterase III inhibitor, cilostazol plays a significant role in hydrolyzing cyclic adenosine monophosphate (cAMP) within platelets, by a different mechanism from that of aspirin and clopidogrel [13], which leads to reduced aggregation of platelets. In addition to the drug's antiplatelet function, its upregulation of p53, p21 and hepatocyte growth factor through altering the cAMP levels in vascular smooth muscle cells has an antiproliferative effect [32]. Cilostazol also has antirestenosis effects by inhibiting stent-induced P-section expression in platelets and upregulating leukocyte Mac-1, which triggers restenosis after PCI surgery [33], possibly explaining the reduced rates of TLR and TVR. TAT group patients benefited from a lower incidence of MACEs, mainly driven by reduced repeat revascularization. Rizzo et al. [34] found that cilostazol improved the pro-atherogenic lipid profile in patients with type 2 diabetes. In addition, Ahn et al. [35] demonstrated that TAT had a better anti-inflammatory effect than DAT, which may be another mechanism benefitting MACEs. However, in that meta-analysis, TAT failed to reduce the incidence of stent thrombosis because the small sample size was underpowered to detect a significant difference between TAT and DAT due to the low incidence of stent thrombosis in the real world.

The clinical benefit of intensified antiplatelet therapy may be offset by an associated increase in bleeding complications; however, when cilostazol was administered as an adjunctive to aspirin and clopidogrel, the intensified antiplatelet effect could lead to a lessened HTPR, but did not increase the risk of bleeding events, which was demonstrated in previous meta-analyses and was consistent with all the included 
Citation: Chen HB, Zhang X, Liang H, Liu X, Xiu J (2015) Cilostazol Added to Dual Antiplatelet Therapy for Patients with High Risk of Restenosis after Drug-Eluting Stent Implantation: A Systematic Review and Meta-Analysis of RCTs. Cardiol Pharmacol 4: 133. doi:10.4172/2329-6607.1000133

Page 9 of 11

\begin{tabular}{|c|c|c|c|c|c|}
\hline & Declare-Long II & Declare-Long & Youn et al. & Declare-Diabetes & Gao et al. \\
\hline Study design & RCT & RCT & RCT & RCT & RCT \\
\hline Country & South Korea & South Korea & South Korea & South Korea & China \\
\hline Numbers of centers & 10 & 5 & 18 & 5 & 1 \\
\hline Follow-up period $(\mathrm{m})$ & 12 & 9 & 12 & 6 & 12 \\
\hline Follow-up proportion (\%) & 100 & 100 & 100 & 100 & 100 \\
\hline Numbers of patients (n) & TAT/DAT $=250 / 249$ & TAT/DAT $=250 / 250$ & TAT/DAT $=308 / 307$ & TAT/DAT $=200 / 200$ & TAT/DAT $=213 / 215$ \\
\hline Age (years) & TAT/DAT $=61 / 62$ & TAT/DAT=60.9/61.2 & TAT/DAT $=64 / 65$ & TAT/DAT $=61 / 61$ & TAT/DAT $=58 / 53$ \\
\hline Male (\%) & TAT/DAT=70/71.5 & TAT/DAT=64.8/63.6 & TAT/DAT $=64.2 / 63$ & TAT/DAT $=59 / 57$ & TAT/DAT=78.9/81.9 \\
\hline Hypertension (\%) & TAT/DAT $=58.4 / 64.7$ & TAT/DAT $=54.8 / 55.2$ & TAT/DAT $=65.8 / 68.2$ & TAT/DAT $=59.5 / 59.5$ & TAT/DAT $=56.3 / 54.4$ \\
\hline Smoking (\%) & TAT/DAT $=30.4 / 30.1$ & TAT/DAT=37.6/37.2 & TAT/DAT $=44 / 48.4$ & TAT/DAT $=24 / 31.5$ & TAT/DAT $=38.5 / 42.3$ \\
\hline Diabetes mellitus (\%) & TAT/DAT=36.8/33.7 & TAT/DAT $=34 / 32.4$ & TAT/DAT $=30.9 / 32.5$ & TAT/DAT $=100 / 100$ & TAT/DAT $=19.2 / 16.2$ \\
\hline LCL (\%) & TAT/DAT=100/100 & TAT/DAT $=100 / 100$ & NA & NA & NA \\
\hline Lesion length $(\mathrm{mm})$ & $\mathrm{TAT} / \mathrm{DAT}=33.6 / 34.7$ & $\geq 25$ & $\geq 28$ & TAT/DAT=26.7/26.3 & NA \\
\hline Stent length (mm) & TAT/DAT=37.3/39.1 & $\geq 32$ & TAT/DAT $=30.4 / 30.8$ & TAT/DAT=33.5/32.1 & NA \\
\hline Multivessel disease (\%) & TAT/DAT $=34.8 / 37.3$ & TAT/DAT=66.8/58.6 & TAT/DAT $=65.8 / 73.4$ & TAT/DAT=65.5/62.5 & TAT/DAT $=91.1 / 78.6$ \\
\hline Multivessel stenting (\%) & TAT/DAT $=34.8 / 37.3$ & $\mathrm{TAT} / \mathrm{DAT}=44.8 / 37.2$ & TAT/DAT $=53.4 / 58.1$ & TAT/DAT $=36.5 / 30$ & NA \\
\hline \multicolumn{6}{|l|}{ Clinical diagnosis } \\
\hline Stable angina (\%) & TAT/DAT=44/50.6 & $\mathrm{TAT} / \mathrm{DAT}=47.2 / 43.6$ & TAT/DAT $=35.5 / 34.7$ & TAT/DAT=41.5/42.5 & NA \\
\hline Unstable angina (\%) & TAT/DAT $=44.8 / 40.2$ & TAT/DAT=34/36.4 & TAT/DAT=41.4/46.8 & TAT/DAT=38/35.5 & NA \\
\hline AMI (\%) & TAT/DAT $=11.2 / 9.2$ & NA & TAT/DAT $=20.2 / 16.6$ & TAT/DAT $=20.5 / 22$ & NA \\
\hline LVEF $(\%)$ & TAT/DAT $=60.7 / 59.8$ & TAT $/$ DAT $=59 / 58$ & TAT/DAT $=53.3 / 57.6$ & TAT $/$ DAT $=59 / 58$ & TAT/DAT $=50 / 48.6$ \\
\hline Aspirin (mg) & 200 (loading and qd) & 200 (daily) & 300 (loading)+100(qd) & $200(q d)$ & $100(q d)$ \\
\hline Clopidogrel (mg) & 300 (loading)+75(qd) & 300 (loading)+75 (qd) & $300-600$ (loading)+75(qd) & 300 (loading)+75 (qd) & 75 (qd) \\
\hline Cilostazol (mg) & 200 (loading and qd) & $200(q d)$ & 200 (loading)+100(bid) & 200 (daily) & $200(q d)$ \\
\hline Duration of DAT $(\mathrm{m})$ & 12 & 9 & 12 & 6 & 12 \\
\hline Duration of cilostazol $(\mathrm{m})$ & 8 & 6 & 3 & 6 & 12 \\
\hline
\end{tabular}

Notes: RCT=Randomized Controlled Trial; TAT=cilostazol based Triple Antiplatelet Therapy; DAT=Dual Antiplatelet Therapy (aspirin+ clopidogrel); LCL= Long Coronary Lesion; AMI=Acute Myocardial Infarction; LVEF=Left Ventricular Ejection Fraction; $m=$ months; qd=once a day; bid=twice a day; NA= Not Available Table1: Characteristics of included studies

\begin{tabular}{|c|c|c|c|c|c|}
\hline & Declare-Long II & Declare-Long & Youn et al. & Declare-Diabetes & Gao et al. \\
\hline Trials described as randomized & Yes & Yes & Yes & Yes & Yes \\
\hline Trial described method to generate randomization & Yes & Yes & Yes & Yes & No \\
\hline Trials described as double blind & Yes & No & No & No & No \\
\hline Adequate description of double blinding method & Yes & No & No & No & No \\
\hline Description of withdrawal and dropout & Yes & Yes & Yes & Yes & Yes \\
\hline Concealed treatment allocation & $\mathrm{CC}$ & $\mathrm{CC}$ & $\mathrm{CC}$ & $\mathrm{CC}$ & UC \\
\hline Sum of Jadad score & 7 & 5 & 5 & 5 & 2 \\
\hline
\end{tabular}

Notes: $\mathrm{CC}=$ Clearly Concealed; UC= Unclearly Concealed

Table 2: Quality of included studies

\begin{tabular}{|l|c|c|}
\hline & Begg's test $(\mathbf{p})$ & Egger's test $(\mathbf{p})$ \\
\hline All-cause mortality & 0.462 & 0.252 \\
\hline MACEs & 1.000 & 0.073 \\
\hline Bleeding & 0.462 & 0.111 \\
\hline Stent thrombosis & 0.462 & 0.335 \\
\hline In-stent late loss & 0.117 & 0.129 \\
\hline In-segment late loss & 0.602 & 0.404 \\
\hline TLR & 0.806 & 0.968 \\
\hline TVR & 0.462 & 0.583 \\
\hline In-stent restenosis & 0.117 & \\
\hline In-segment restenosis & 0.602 & \\
\hline Other adverse reactions & 0.086 & \\
\hline Drug discontinuation & 0.117 & \\
\hline
\end{tabular}

MACEs= Major Adverse Events; TLR= Target Lesion Revascularization;

TVR= Target Vessel Revascularization

Table3: Begg's and Egger's tests for publication bias 
trials comparing TAT versus DAT in this meta-analysis. However, we cannot ignore the higher incidence of other adverse reactions, such as rash, gastrointestinal trouble, thrombocytopenia, neutropenia, hepatic dysfunction, and headache, which could lead to more drug discontinuation in patients after DES implantation compared with DAT.

Currently, new generation ADP-receptor antagonists, such as prasugrel and ticagrelor, have received great attention, because large RCTs such as PLATO [36] and ATLANTIC [37], demonstrated that prasugrel or ticagrelor was superior to clopidogrel for the prevention of ischemic events in ACS patients who accepted PCI treatment. Further studies are needed in this area, which will be helpful in updating future antiplatelet treatment strategies for reducing the high risk of restenosis after DES implantation.

Our study has potential limitations. First, only five RCTs were involved in this meta-analysis, and most of the subjects enrolled were from South Korea. Thus, caution should be taken when generalizing the findings to more diverse patient populations. Further studies in this field should be performed to determine whether similar clinical results can be achieved in different ethnic groups, especially in Western populations. Second, only Declare-Long II was double-blinded, so further high quality articles are warranted to perform in this field. Finally, we limited our analysis to RCTs only. The Generalization of our findings could be improved by including non-randomized designs such as cohort and case-control studies, which may increase the risk of bias and heterogeneity that could make data interpretation more difficult.

\section{Conclusions}

Compared with DAT, patients with a high risk of restenosis benefited from TAT in reduced stent restenosis and revascularization after DES implantation, without increases in all-cause mortality and bleeding, but these outcomes were accompanied by a higher incidence of other adverse reactions and drug discontinuation.

\section{Acknowledgements}

This work was supported by a grant from the National Nature Science Funding of China (81470598).

\section{References}

1. Jneid H, Anderson JL, Wright RS, Adams CD, Bridges CR, et al. (2012) 2012 ACCF/AHA focused update of the guideline for the management of patients with unstable angina/Non-ST-elevation myocardial infarction (updating the 2007 guideline and replacing the 2011 focused update): a report of the American College of Cardiology Foundation/American Heart Association Task Force on practice guidelines. Circulation 126: 875-910.

2. Kushner FG, Hand M, Smith SC Jr, King SB 3rd, Anderson JL, et al. (2009) 2009 focused updates: ACC/AHA guidelines for the management of patients with ST-elevation myocardial infarction (updating the 2004 guideline and 2007 focused update) and ACC/AHA/SCAI guidelines on percutaneous coronary intervention (updating the 2005 guideline and 2007 focused update) a report of the American College of Cardiology Foundation/American Heart Association Task Force on Practice Guidelines. J Am Coll Cardiol 54: 2205-2241.

3. Parodi G, Marcucci R, Valenti R, Gori AM, Migliorini A, et al. (2011) High residual platelet reactivity after clopidogrel loading and long-term cardiovascular events among patients with acute coronary syndromes undergoing PCI. JAMA 306: 1215-1223.

4. Gurbel PA, Bliden KP, Hiatt BL, O'Connor CM (2003) Clopidogrel for coronary stenting: response variability, drug resistance, and the effect of pretreatment platelet reactivity. Circulation 107: 2908-2913.

5. Wang ZJ, Zhou YJ, Liu YY, Yu M, Shi DM, et al. (2009) Impact of clopidogrel resistance on thrombotic events after percutaneous coronary intervention with drug-eluting stent. Thromb Res 124: 46-51.
6. Sofi F, Marcucci R, Gori AM, Abbate R, Gensini GF (2008) Residual platelet reactivity on aspirin therapy and recurrent cardiovascular events--a metaanalysis. Int J Cardiol 128: 166-171.

7. Lee CW, Park DW, Lee BK, Kim YH, Hong MK, et al. (2006) Predictors of restenosis after placement of drug-eluting stents in one or more coronary arteries. Am J Cardiol 97: 506-511.

8. Wang ZJ, Zhou YJ, Liu YY, Yu M, Shi DM, et al. (2009) Obesity and cardiovascular thrombotic events in patients undergoing percutaneous coronary intervention with drug-eluting stents. Heart 95: 1587-1592.

9. Angiolillo DJ, Fernández-Ortiz A, Bernardo E, Barrera Ramírez C, Sabaté M, et al. (2004) Platelet aggregation according to body mass index in patients undergoing coronary stenting: should clopidogrel loading-dose be weight adjusted? J Invasive Cardiol 16: 169-174.

10. Hong SJ, Kim MH, Ahn TH, Ahn YK, Bae JH, et al. (2006) Multiple predictors of coronary restenosis after drug-eluting stent implantation in patients with diabetes. Heart 92: 1119-1124.

11. Pan X, Arauz E, Krzanowski JJ, Fitzpatrick DF, Polson JB (1994) Synergistic interactions between selective pharmacological inhibitors of phosphodiesterase isozyme families PDE III and PDE IV to attenuate proliferation of rat vascular smooth muscle cells. Biochem Pharmacol 48: 827-835.

12. Douglas JS Jr (2007) Pharmacologic approaches to restenosis prevention. Am J Cardiol 100: 10K-6K

13. Goto S (2005) Cilostazol: potential mechanism of action for antithrombotic effects accompanied by a low rate of bleeding. Atheroscler Suppl 6: 3-11.

14. Geng DF, Deng J, Jin DM, Wu W, Wang JF (2012) Effect of cilostazol on the progression of carotid intima-media thickness: a meta-analysis of randomized controlled trials. Atherosclerosis 220: 177-183.

15. Sakurai R, Koo BK, Kaneda H, Bonneau HN, Nagai R (2013) Cilostazol added to aspirin and clopidogrel reduces revascularization without increases in major adverse events in patients with drug-eluting stents: a meta-analysis of randomized controlled trials. Int J Cardiol 167: 2250-2258.

16. Takagi H, Umemoto $\mathrm{T}$ (2011) Benefit, rather than safety, of cilostazol for longterm mortality in patients undergoing percutaneous coronary intervention: a meta-analysis of randomized trials. Int J Cardiol 153: 74-76.

17. Chen Y, Zhang Y, Tang Y, Huang X, Xie Y (2014) Long-term clinical efficacy and safety of adding cilostazol to dual antiplatelet therapy for patients undergoing $\mathrm{PCl}$ : a meta-analysis of randomized trials with adjusted indirect comparisons. Curr Med Res Opin 30: 37-49.

18. Gao W, Zhang Q, Ge H, Guo Y, Zhou Z (2013) Efficacy and safety of triple antiplatelet therapy in obese patients undergoing stent implantation. Angiology 64: 554-558.

19. Lee SW, Lee JY, Ahn JM, Park DW, Han S, et al. (2013) Comparison of dua versus triple antiplatelet therapy after drug-eluting stent according to stent length (from the pooled analysis of DECLARE trials). Am J Cardiol 112: 17381744.

20. Youn YJ, Lee JW, Ahn SG, Lee SH, Choi H, et al. (2014) Multicenter randomized trial of 3-month cilostazol use in addition to dual antiplatelet therapy after biolimus-eluting stent implantation for long or multivessel coronary artery disease. Am Heart J 167: 241-248.

21. Moher D, Liberati A, Tetzlaff J, Altman DG; PRISMA Group (2010) Preferred reporting items for systematic reviews and meta-analyses: the PRISMA statement. Int J Surg 8: 336-341.

22. Jadad AR, Moore RA, Carroll D, Jenkinson C, Reynolds DJ, et al. (1996) Assessing the quality of reports of randomized clinical trials: is blinding necessary? Control Clin Trials 17: 1-12.

23. Detsky AS, Naylor CD, O'Rourke K, McGeer AJ, L'Abbé KA (1992) Incorporating variations in the quality of individual randomized trials into meta-analysis. J Clin Epidemiol 45: 255-265.

24. Galbraith RF (1988) A note on graphical presentation of estimated odds ratios from several clinical trials. Stat Med 7: 889-894.

25. Begg CB, Mazumdar M (1994) Operating characteristics of a rank correlation test for publication bias. Biometrics 50: 1088-1101

26. Egger M, Davey Smith G, Schneider M, Minder C (1997) Bias in meta-analysis detected by a simple, graphical test. BMJ 315: 629-634. 
Citation: Chen HB, Zhang X, Liang H, Liu X, Xiu J (2015) Cilostazol Added to Dual Antiplatelet Therapy for Patients with High Risk of Restenosis after Drug-Eluting Stent Implantation: A Systematic Review and Meta-Analysis of RCTs. Cardiol Pharmacol 4: 133. doi:10.4172/2329-6607.1000133

27. Duval S, Tweedie R (2000) Trim and fill: A simple funnel-plot-based method of testing and adjusting for publication bias in meta-analysis. Biometrics 56 : $455-463$

28. Lee SW, Park SW, Kim YH, Yun SC, Park DW, et al. (2007) Comparison of triple versus dual antiplatelet therapy after drug-eluting stent implantation (from the DECLARE-Long trial). Am J Cardiol 100: 1103-1108.

29. Lee SW, Park SW, Kim YH, Yun SC, Park DW, et al. (2008) Drug-eluting stenting followed by cilostazol treatment reduces late restenosis in patients with diabetes mellitus the DECLARE-DIABETES Trial (A Randomized Comparison of Triple Antiplatelet Therapy with Dual Antiplatelet Therapy After Drug-Eluting Stent Implantation in Diabetic Patients). J Am Coll Cardiol 51: 1181-1187.

30. Lee SW, Park SW, Kim YH, Yun SC, Park DW, et al. (2011) A randomized, double-blind, multicenter comparison study of triple antiplatelet therapy with dual antiplatelet therapy to reduce restenosis after drug-eluting stent implantation in long coronary lesions: results from the DECLARE-LONG II (Drug-Eluting Stenting Followed by Cilostazol Treatment Reduces Late Restenosis in Patients with Long Coronary Lesions) trial. J Am Coll Cardiol 57: 1264-1270.

31. Levine GN, Bates ER, Blankenship JC, Bailey SR, Bittl JA, et al. (2011) 2011 ACCF/AHA/SCAI Guideline for Percutaneous Coronary Intervention: executive summary: a report of the American College of Cardiology Foundation/American Heart Association Task Force on Practice Guidelines and the Society for Cardiovascular Angiography and Interventions. Circulation 124: 2574-2609.
32. Morishita R (2005) A scientific rationale for the CREST trial results: evidence for the mechanism of action of cilostazol in restenosis. Atheroscler Suppl 6: $41-46$

33. Inoue T, Uchida T, Sakuma M, Imoto Y, Ozeki Y, et al (2004) Cilostazol inhibits leukocyte integrin Mac-1, leading to a potential reduction in restenosis after coronary stent implantation. J Am Coll Cardiol 44: 1408-1414.

34. Rizzo M, Corrado E, Patti AM, Rini GB, Mikhailidis DP (2011) Cilostazol and atherogenic dyslipidemia: a clinically relevant effect? Expert Opin Pharmacother 12: 647-655.

35. Ahn CM, Hong SJ, Park JH, Kim JS, Lim DS (2011) Cilostazol reduces the progression of carotid intima-media thickness without increasing the risk of bleeding in patients with acute coronary syndrome during a 2-year follow-up. Heart Vessels 26: 502-510.

36. James S, Akerblom A, Cannon CP, Emanuelsson H, Husted S, et al. (2009) Comparison of ticagrelor, the first reversible oral $\mathrm{P} 2 \mathrm{Y}(12)$ receptor antagonist, with clopidogrel in patients with acute coronary syndromes: Rationale, design, and baseline characteristics of the PLATelet inhibition and patient Outcomes (PLATO) trial. Am Heart J 157: 599-605.

37. Montalescot G1, van 't Hof AW, Lapostolle F, Silvain J, Lassen JF, et al. (2014 Prehospital ticagrelor in ST-segment elevation myocardial infarction. N Engl J Med 371: 1016-1027. 